Ann. Sci. forest., 1980, 37 (4), 375-380.

\title{
Compte rendu des excursions en forêt
}

\author{
R. PERRIN \\ Loboratoire de Pathologie forestière, \\ Centre national de Recherches forestières, I.N.R.A., \\ Champenoux 54280 Seichamps
}

\section{Visite d'un peuplement de hêtres en forêt d'Amance}

La forêt d'Amance s'étend sur 1100 ha, à 10 km au Nord-Est de Nancy (Meurtheet-Moselle).

Les sols, développés sur limons recouvrant les marnes argileuses du Rhétien supérieur, conviennent bien au Chêne qui prédomine. Par place cependant, le hêtre forme des peuplements, là où affleure le grès infraliasique, notamment en parcelle 41 , où la maladie de l'écorce du hêtre sévit dans la hêtraie (80-100 ans) installée sur un sol brun lessivé à marmorisation profonde. Le dispositif d'observation établi depuis 4 ans fut un bon prétexte à la confrontation des idées et au débat, sur différents aspects de ce phénomène parasitaire.

Les populations de cochenille sont globalement en lente progression depuis 1975. Les fluctuations constatées sur certains arbres sont parfois très marquées. La localisation préférentielle des colonies de cochenilles sur la face abritée du ruissellement de l'eau sur le tronc des arbres inclinés, est la seule liaison flagrante avec les facteurs de l'environnement.

Les premiers signes extérieurs de la présence de Nectria coccinea dans les tissus corticaux étaient particulièrement évidents sur quelques arbres. Faute de pouvoir se nourrir dans l'écorce altérée par $N$. coccinea, la cochenille régresse et n'assure plus la production de nouvelles cires. Les formations anciennes noircissent, délimitant une zone contrastant avec l'aspect blanchâtre de l'écorce là où Cryptococcus fagi persiste.

Ce symptôme précède de peu l'apparition d'un suintement souvent abondant au printemps ou à l'automne, accompagné de la différenciation du stade imparfait Cylindrocarpon candidum, en périphérie de la zone altérée. Les sporodochies, blanches, se confondent souvent avec les cires produites par l'insecte.

A partir de ce stade le déclin de l'arbre est sujet à des variations de trois ordres :

\section{Individuelles}

Certains arbres périssent en quelques mois, parfois à la suite d'un brusque flétrissement estival. D'autres, malgré de multiples suintements, s'opposent à l'extension des nécroses corticales par une active cicatrisation. Leur dépérissement dure plusieurs années, ou quelquefois ils se rétablissent, mais l'écorce conserve de longues 
années un aspect tourmenté, vestige d'anciennes lésions. De telles différences dans le comportement individuel des arbres suggèrent, parmi d'autres, un déterminisme génétique à la tolérance, voire à la résistance de certains arbres à cette maladie.

\section{Locales}

Dans une même forêt, ou un territoire homogène sur le plan macroclimatique, la gravité de la maladie dépend des conditions stationnelles. Ainsi en Lorraine, la manifestation épidémique de la maladie de l'écorce du hêtre est, à l'heure actuelle, limitée aux hêtraies établies sur des sols bruns lessivés à pseudogley. L'importance je la maladie est également liée à l'âge des arbres. Bénigne chez les jeunes hêtres, elle occasionne de graves préjudices aux hêtraies parvenues à maturité, ou surannées.

\section{Régionales}

Le délai écoulé entre l'apparition du suintement ef la mort de l'arbre oppose les hêtraies normandes et picardes, où il est très court, aux hêtraies du Nord-Est où il peîl atteindre plusieurs années. L'insuffisance de nos connaissances actuelles ne permet pas de faire la part entre influence climatique et effet provenance.

Le symptôme ultérieur résulte du dessèchement de l'écorce détruite par M. coccinea. De larges fissures annoncent le décollement de l'écorce par larges plaques, porteuses d'une multifude de périthèces de $N$. coccinea. C'est dans cet état que les congressistes onł trouvé les arbres qui, l'année précédente, hébergeaient Gonatorhodiella highlei (Nematogenium ferrugineum) hyperparasite de $N$. coccinea (première mention européenne). Cette évolution fatale révèle l'impuissance d'une lutte biologique que certains estimaient prometteuse. L'intervention de G. highlei est à la fois beaucoup trop tardive, et imparfaite. Les participants ont alors évoqué et débaltu d'autres procédés de lutte biologique, comme l'influence de la flore épithytique sur les pullulations de la cochenille. Il est bien établi maintenant que la présence d'Ascodichaena rugosa, ascomycète très commun sur l'écorce du hêtre, contrarie l'installation de la cochenille. L'exploitation de ce phénomène sur le plan pratique paraît malaisée. D'autres épiphytes, au contraire, favorisent les pullulations de la cochenille. Un lichen commun, Lecanora conizaeoides, réalise des niches écologiques en formant par sa rugosité des abris, facilitant l'installation et le développement des colonies de C. fagisuga. Enfin, les prédateurs de la cochenille, principalement plusieurs espèces do coccinelles, ont semble-t-il peu d'influence sur ses pullulations.

La lutte biologique se révèle infructueuse au stade actuel des connaissances, cependant imparfaites.

A partir d'un premier arbre dépérissant (1974), totalement altéré par les orgarismes secondaires (xylophages et lignivores), brisé sous l'effet du vent, les congressistes ont pu apprécier le processus d'extension de la maladie en taches d'huile. II trouve son explication daris une dispersion par le vent à partir du premier arbre ctteint. L'unanimité s'est faite sur la nécessité d'une intervention précoce lors des coupes sanitaires. L'abattage de l'arbre, au stade où la cochenille pullule (revêtement blanc), avant même l'apparition d'un suintement, préserve la valeur marchande de l'arbre. En infervenant avant la différenciation des périthèces, on réduit considérablement la quantité d'ascospores produites et, par conséquent, les risques 
d'infection diminuent. La contrepartie, cependant inévitable, à cette intervention, est la création de larges ouvertures dans les peuplements, sources de nombreuses complications pour la gestion. L'écorce des arbres voisins, brutalement exposée à la lumière, peut être victime d'insolation ou d'excès de température, entraînant parfois la mort, ou accentuant les effets de la maladie.

Enfin, les participants ont pu considérer l'évolution de la nécrose de l'écorce du hêtre artificiellement inoculée avec $N$. coccinea. La part déterminante assumée par la cochenille dans l'évolution des nécroses est particulièrement évidente à la suite de cette étude expérimentale.

L'abondance du chancre du hêtre (Nectria ditissima tul.) a frappé les participants, qui, en plus des symptômes caractéristiques, ont reconnu l'influence manifeste des arbres semenciers sur la propagation de la maladie.

\section{Visite de la forêt de Retz (Aisne et Oise)}

La forêt a une superficie totale de 13000 ha où le Hêtre est l'essence dominante (45 p. 100). Elle est assise sur des terrains tertiaires (lutécien) recouverts sur plus des deux tiers par les sable et grès de Beauchamp (Auversien). La gamme de sols est très vaste, allant du sol brun lessivé sain ou plus ou moins hydromorphe, jusqu'au podzol typique.

Le service forestier local a présenté le type de dispositif adoplé pour suivre l'évolution de la maladie de l'écorce du hêtre. De nombreuses placettes de trente arbres (tache) sont dispersées dans toute la forêt, occupant des situations très variées (âge, conditions stationnelles, densité des tiges, mélange). Tous les 3 mois environ, le chef de triage crédite les arbres d'une note prenant en compte l'abondance de la cochenille, la présence de Nectria coccinea (suintement), et l'état d'avancement du dépérissement.

En plus de l'intérêt éducalif évident, le dispositif permet une perception objective de l'évolution de la maladie, en fonction des diverses situations. Une première compa. raison a pu être établie entre avril 1978 et mai 1979, indiquant une nette progression de la cochenille, el corrélativement des arbres infectés par Nectria coccinea. Un déficit pluviométrique, el par conséquent l'absence de lessivage lors de la phase mobile de l'insecte, pourrait être à l'origine de cette recrudescence de Cryptococcus fagisuga, que les basses températures hivernales ont insuffisamment freinée.

L'échelle de notation diffère notablement de celles choisies par les scientifiques, el la comparaison des résultats acquis dans cette forêt devient difficile. Malgré le nombre élevé de placettes, la multitude des facteurs de variations, interférant les uns avec les autres, limite considérablement les chances de révéler leur influence. Néanmoins, chacun a reconnu l'immense intérêf de cette initiative qui mérite d'être étendue, après que les objectifs à atteindre et surtout une échelle de notation standard auront été définis.

\section{Références bibliographiques}

Notice sur la forêt domaniale de Retz. Station de Sylviculfure du C.N.R.F., 1i p., révisée par H. Osvald en avril 1979.

Etude sur l'évolution du Cryptococcus fagi et du Nectria. Office National des Forêts. Région Picardia. Forêt de Retz. 1 p., mai 1979. 


\section{Tournée en forêt domaniale de Lyons (Eure et Seine-Maritime)}

Monsieur Mormiche, Directeur Régional de l'Office des Forêts, pour la Normandie, a brossé un tableau très complet de la hêtraie normande et des préjudices que le dépérissement lui fait subir depuis 1970.

La forêt domaniale de LYONS, réputée être la plus belle hêtraie de France (10 $673 \mathrm{ha}$ ), est le plus grand des massifs forestiers de Normandie (Eure et SeineMaritime) où la superficie des forêts domaniales atteint 37000 ha. La « hêtraie normande » est caractérisée par un traitement en futaie régulière, dont l'essence principale presque exclusive est le hêtre. Cette hêtraie essentiellement artificielle diffère sensiblement des associations climaciques des différentes stations sur lesquelles l'homme l'a imposée. Après les prélèvements excessifs ef incontrôlés entre 1790 et 1826, le forestier a entrepris une conversion en futaie régulière avec pour objectif, le hêtre, choisi pour différentes raisons : «pillage» du chêne pour la construction et la marine, exportation du hêtre vers l'Angleterre qui lui vaut aujourd'hui encore les cours les plus élevés de France, réussite d'ensemencements naturels au début du $\mathrm{XIX}$ siècle.

Le climat à dominante océanique dégradée, doux el humide, convient parfaitement au hêtre. L'assise géologique, la craie sénonienne affleurant au niveau des versants, est surmontée d'une couche d'argile à silex. Le limon repose sur cette argile occupant la totalité des plateaux $(80$ p. 100), où l'on peut définir deux types de stations sur sols lessivés ef podzoliques :

- la chênaie-hêtraie mésotrophe à charme.

- La chênaie-hêtraie acidophile à houx.

Enfin sur les versants crayeux, la hêtraie calcicole se rencontre sur des sols plus ou moins carbonatés, seule station favorable à une régénération naturelle du hêtre.

Le prélèvement moyen annuel est environ de $7 \mathrm{~m}^{3} / \mathrm{ha} / \mathrm{an}$.

Une journée entière a été consacrée à la visite de dispositifs expérimentaux, ou d'observations, et de parcelles illustrant l'incidence de la maladie sur la hêtraie.

\section{1. - Dispositifs d'observations de la maladie}

Fruit de l'étroite collaboration de I'INRA et de l'ONF, l'expérience mise en place dans les trois principales hêtraies normandes (Lyons, Eawy, Eu) est destinée à suivre l'évolution de la maladie de l'écorce du hêtre. En forêt de Lyons, les neuf placettes implantées résultent de la combinaison de trois stations (limon, limon à silex, et craie) et de trois classes d'âge (70 à 95 ans, 96 à 120 ans et + de 150 ans). De forme rectangulaire, les placettes ont une longueur de $200 \mathrm{~m}$, et comprennent toujours cent arbres numérotés (transect), qui sont l'objet d'une observation individuelle. Chaque année, en octobre, l'abondance de l'insecte et l'importance des nécroses dues à Nectria coccinea, sont appréciées à l'aide d'une échelle de notation universellement adoptée par les scientifiques.

Le premier enseignement de ce dispositif est la confirmation d'une moindre sévérité de la maladie sur les' pentes calcaires en forêf de Lyons ; elle est pratiquement 
absente de la parcelle CF 1 , où la hêtraie âgée de 180 ans (sur craie) a un volume sur pied de $424 \mathrm{~m}^{3} / \mathrm{ha}$ ). La jeune futaie ( 85 ans) sur limon ( $\mathrm{P} \mathrm{A}_{2} 8^{\mathrm{e}}$ série) est au contraire l'objet d'une forte infestation de cochenilles, ef le préjudice subi, qui s'accumule depuis 1970 est considérable.

Les participants ont alors établi la comparaison avec le dispositif adopté en forêt de Retz, et entamé une discussion sur l'intérêt et la conception des dispositifs d'observation.

Le système du transect est celui qui semble le mieux adapté à la maladie de l'écorce du hêtre, à condition de définir avec précision l'objectif à atteindre. Certains participants souhaitent vivement la création de ce type de dispositifs dans plusieurs pays européens, où seraient effectuées des observations standardisées, comparables, d'où se dégageraient les influences de différents facteurs (stationnels ef sylvicoles).

\section{2. - Etude expérimentale de l'influence de l'éclaircie sur la maladie} de l'écorce du hêtre

Les nombreuses observations, souvent contradictoires, ne permettent pas de connaître l'influence de l'éclaircie sur le dépérissement du hêtre. Seul un dispositif expérimental, conçu spécialement dans ce but, pouvait combler cette lacune (collaboration R. Perrin, H. Oswald).

Les placettes unitaires, carrées, ont une superficie de 0,25 ha, entourée d'un périmètre de protection ( $10 \mathrm{~m}$ de large), traitée de la même manière que la placette où se font les observations. Après inventaire, et par référence aux tables de production anglaises, on a défini différentes normes d'intervention (soixante, cent, cent cinquante, deux cents arbres à l'ha à terme). Les placettes (vingt-deux) sont réparties dans cinq parcelles, en combinant trois classes d'âge (40-70 ans, 70-95 ans, 96-120 ans), et plusieurs niveaux d'éclaircies effectuées soit au profit d'arbre de place, soit «par le bas ».

L'évolution des populations de la cochenille, l'apparition et l'extension des lésions corticales engendrées par $N$. coccinea seront appréciées par observation individuelle de tous les arbres de la placette, ou d'un large échantillon, au mois d'octobre de chaque année.

3. - Surveillance expérimentale de l'évolution de la maladie de l'écorce du hêtre, à l'aide de la photographie aérienne infra-rouge couleur

Les documents acquis, à la suite de la première mission effectuée en 1975, ont permis la répartition des arbres de la parcelle $C_{6}$, 3 e série (155 ans) en cinq classes selon leur réflectance. Les arbres morts apparaissent en bleu vert. Cent quatre-vingts arbres ont pu être repérés individuellement, distribués dans les quatre autres classes caractérisées par des couleurs variant du rose pâle verdâtre au rose pâle, puis au rose, et enfin au rouge là où figurent les rares chênes de la parcelle.

Au printemps ef à l'automne de chaque année les arbres sont décrits à travers de nombreux critères (débourrement, analyse foliaire, turgescence foliaire, turgescence corticale, infestation de cochenilles, nécrose corticale, présence de $N$. coccinea, sénescence foliaire, organisme secondaire, aptitude à la cicatrisation, ...). 
Les hêtres, à faible réflectance, débourrent plus tardivement, ont un feuillage jaunissant plus précocement, et montrent une aptitude à la cicatrisation plus faible que les autres. La liaison est parfaite entre la réflectance et l'état sanitaire de l'arbre. Cette étude révèle l'influence de facteurs indéterminés sur la physiologie de l'arbre, qui devient plus vulnérable aux infestations de la cochenille. Cette prédisposition des arbres à l'insecte comme au champignon, suggérée également par d'autres études, est, de l'avis général, une phase fondamentale du phénomène maladif, intervenant à la fois dans le déclenchement, et l'ampleur de la maladie.

\section{4. - La maladie de l'écorce du hêtre en taillis sous futaie}

L'existence d'un foyer, très localisé, dans une parcelle traitée en taillis sous futaie, indique que cette maladie n'est pas cantonnée à la futaie régulière, qui subit, cependant, les préjudices les plus élevés.

A l'issue de cette journée, l'impression des participants, ressort parfaitement dans les conclusions de Monsieur Mormiche, Directeur Régional de l'O. N. F., pour la Normandie : «La hêtraie normande, monoculture artificielle, imposée par l'homme a élé adoptée par les hommes comme faisant partie du patrimoine naturel de leur région.

Au plan économique, la conversion du XIX $X^{e}$ siècle s'avère une brillante réussite. Malheureusement les échecs de la première moitié du $\mathrm{xx}^{\mathrm{e}}$ siècle et les difficultés rencontrées actuellement ne préparent pas des résultats aussi favorables. Au plan cultural, voire écologique, l'échec s'installe (régénération naturelle impossible, chancre et dépérissement du hêtre, chablis...).

L'histoire de la conversion menée depuis le début du XIX ${ }^{e}$ siècle en Normandie eł ses résultats méritent sûrement une réflexion approfondie. »

Reçu pour publication en décembre 1979.

\section{Références bibliographiques}

MORMICHE A., mai 1979. Les hêtraies normandes, 11 p.

BORDERES M., 1978. Présentation de la forêt de Lyons, 7 p. 